\title{
Tripartite Ramsey Numbers for Paths
}

\author{
András Gyárfás, ${ }^{1}$ Miklós Ruszinkó, ${ }^{1}$ Gábor N. Sárközy, ${ }^{1,2}$ \\ and Endre Szemerédi ${ }^{3}$ \\ ${ }^{1}$ COMPUTER AND AUTOMATION RESEARCH INSTITUTE \\ HUNGARIAN ACADEMY OF SCIENCES \\ BUDAPEST, P.O. BOX 63 \\ BUDAPEST, HUNGARY, H-1518 \\ E-mail: gyarfas@sztaki.hu \\ ruszinko@sztaki.hu \\ ${ }^{2}$ COMPUTER SCIENCE DEPARTMENT \\ WORCESTER POLYTECHNIC INSTITUTE \\ WORCESTER, MASSACHUSETTS, 01609 \\ E-mail:gsarkozy@cs.wpi.edu; \\ gsarkozy@sztaki.hu \\ ${ }^{3}$ COMPUTER SCIENCE DEPARTMENT \\ RUTGERS UNIVERSITY \\ NEW BRUNSWICK, NEW JERSEY, 08903 \\ E-mail: szemered@cs.rutgers.edu
}

Received February 19, 2005; Revised October 20, 2006

Published online 14 February 2007 in Wiley InterScience(www.interscience.wiley.com).

DOI 10.1002/jgt.20231

Abstract: In this article, we study the tripartite Ramsey numbers of paths. We show that in any two-coloring of the edges of the complete tripartite graph $K(n, n, n)$ there is a monochromatic path of length $(1-o(1)) 2 n$. Since $R\left(P_{2 n+1}, P_{2 n+1}\right)=3 n$, this means that the length of the longest monochromatic path is about the same when two-colorings of $K_{3 n}$ and $K(n, n, n)$ are considered. @ 2007 Wiley Periodicals, Inc. J Graph Theory 55: 164-174, 2007

Contract grant sponsor: OTKA (to M.R.); Contract grant numbers: T038198 and T046234; Contract grant sponsor: National Science Foundation (to G.N.S.); Contract grant number: DMS-0456401.

Journal of Graph Theory

(C) 2007 Wiley Periodicals, Inc. 
Keywords: regularity lemma; paths; Ramsey numbers

\section{INTRODUCTION}

\section{A. Ramsey Numbers for Paths}

If $G_{1}$ and $G_{2}$ are graphs, then the Ramsey number $R\left(G_{1}, G_{2}\right)$ is the smallest positive integer $n$ such that if the edges of a complete graph $K_{n}$ are partitioned into 2 disjoint color classes giving graphs $H_{1}$ and $H_{2}$, then one of the subgraphs $H_{i}(i=1,2)$ has a subgraph isomorphic to $G_{i}$. The existence of such a positive integer is guaranteed by Ramsey's original paper [13]. The number $R\left(G_{1}, G_{2}\right)$ is called the Ramsey number for the graphs $G_{1}$ and $G_{2}$. The determination of these numbers has turned out to be remarkably difficult in certain cases (see e.g., [4] or [12] for results and problems). In this article, we consider the case when each $G_{i}$ is a path $P_{n}$ on $n$ vertices. A theorem of Gerencsér and Gyárfás [3] states that

$$
R\left(P_{n}, P_{n}\right)=\left\lfloor\frac{3 n-2}{2}\right\rfloor
$$

Our main result vaguely says that asymptotically this result (perhaps surprisingly) does not change if, instead of a complete graph, a complete graph with "three large holes" (i.e., a balanced tripartite graph) is colored. More precisely:

Theorem 1. In any two-coloring of the edges of the complete tripartite graph $K(n, n, n)$ there is a monochromatic $P_{(1-o(1)) 2 n}$.

We note that recently there has been some interest in tripartite versions of classical results, see e.g., the result of Magyar and Martin [11], a tripartite version of the Corrádi-Hajnal Theorem.

In the proof of Theorem 1 the notion of a connected matching plays a central role; this is a matching $M$ in a graph $G$ such that all edges of $M$ are in the same connected component of $G$. The approach was suggested by Euczak [10] and applied in [2,5].

Sections 2 and 3 provide our main tools including the Regularity Lemma. Then in Section 4, we prove our main lemma (Lemma 7) which states that in any twocoloring of a $(1-\varepsilon)$-dense tripartite graph $G(l, l, l)$ there is a monochromatic connected matching covering almost $2 l$ vertices. Finally in Section 5, we show how Lemma 7 implies Theorem 1.

It is worth noting that Theorem 1 remains true (with the proof of this article) if $o\left(n^{2}\right)$ edges are missing from $K(n, n, n)$. It seems reasonable to conjecture that the following (sharp) version also holds: if the edges of $K(n, n, n)$ are two-colored, then there exists a monochromatic $P_{2 n+1}$ - this would generalize (1) for odd $n$. 


\section{B. Notation and Definitions}

For basic graph concepts see the monograph of Bollobás [1]. Disjoint union of sets will be sometimes denoted by $+V(G)$ and $E(G)$ denote the vertex-set and the edge-set of the graph $G$. Usually, $G_{n}$ is a graph with $n$ vertices, $G(k, k, k)$ is a tripartite graph with $k$-element vertex classes. $(A, B, E)$ denotes a bipartite graph $G=(V, E)$, where $V=A+B$, and $E \subset A \times B . K_{n}$ is the complete graph on $n$ vertices, $K\left(n_{1}, \ldots, n_{k}\right)$ is the complete $k$-partite graph with classes containing $n_{1}, \ldots, n_{k}$ vertices, $P_{n}\left(C_{n}\right)$ is the path (cycle) with $n$ vertices. For a graph $G$ and a subset $U$ of its vertices, $\left.G\right|_{U}$ is the restriction to $U$ of $G$. $\Gamma(v)$ is the set of neighbors of $v \in V$. Hence, the size of $\Gamma(v)$ is $|\Gamma(v)|=\operatorname{deg}(v)=\operatorname{deg}_{G}(v)$, the degree of $v$. $\delta(G)$ stands for the minimum, and $\Delta(G)$ for the maximum degree in $G$. For a vertex $v \in V$ and set $U \subset V-\{v\}$, we write $\operatorname{deg}(v, U)$ for the number of edges from $v$ to $U$. A graph $G_{n}$ is $\gamma$-dense if it has at least $\gamma\left(\begin{array}{l}n \\ 2\end{array}\right)$ edges. $G(k, k, k)$ is $\gamma$-dense if it contains at least $3 \gamma k^{2}$ edges. When $A, B$ are disjoint subsets of $V(G)$, we denote by $e_{G}(A, B)$ the number of edges of $G$ with one endpoint in $A$ and the other in $B$. For non-empty $A$ and $B$,

$$
d_{G}(A, B)=\frac{e_{G}(A, B)}{|A||B|}
$$

is the density of the graph between $A$ and $B$.

Definition 1. The bipartite graph $G=(A, B, E)$ is $(\varepsilon, G)$-regular if

$$
X \subset A, Y \subset B,|X|>\varepsilon|A|,|Y|>\varepsilon|B| \quad \text { imply } \quad\left|d_{G}(X, Y)-d_{G}(A, B)\right|<\varepsilon,
$$

otherwise it is $\varepsilon$-irregular.

\section{THE REGULARITY LEMMA}

In the proof, a two-color version of the Regularity Lemma plays a central role.

Lemma 1 (Regularity Lemma [14]). For every positive $\varepsilon$ and positive integer $m$, there are positive integers $M$ and $n_{0}$ such that for $n \geq n_{0}$ the following holds. For all graphs $G_{1}$ and $G_{2}$ with $V\left(G_{1}\right)=V\left(G_{2}\right)=V,|V|=n$, there is a partition of $V$ into $l+1$ classes (clusters)

$$
V=V_{0}+V_{1}+V_{2}+\cdots+V_{l}
$$

such that

- $m \leq l \leq M$

- $\left|V_{1}\right|=\left|V_{2}\right|=\cdots=\left|V_{l}\right|$

- $\left|V_{0}\right|<\varepsilon n$

- apart from at most $\varepsilon\left(\begin{array}{l}l \\ 2\end{array}\right)$ exceptional pairs, the pairs $\left\{V_{i}, V_{j}\right\}$ are $\left(\varepsilon, G_{s}\right)$-regular for $s=1,2$. 
For an extensive survey on different variants of the Regularity Lemma see [8]. Note also that if we apply the Regularity Lemma for a balanced tripartite graph $G$, we can guarantee that for each cluster that is not $V_{0}$, all vertices of the cluster belong to the same partite class of $G$ (see e.g., [11]).

We will also use the following simple property of $(\varepsilon, G)$-regular pairs.

Lemma 2. Let $G$ be a bipartite graph with bipartition $V(G)=V_{1} \cup V_{2}$ such that $\left|V_{1}\right|=\left|V_{2}\right|=m \geq 45$. Furthermore, let $e_{G}\left(V_{1}, V_{2}\right) \geq m^{2} / 4$ and the pair $\left\{V_{1}, V_{2}\right\}$ be $(\varepsilon, G)$-regular for $0<\epsilon<0.01$. Then for every pair of vertices $v^{\prime}, v^{\prime \prime} \in V(G)$, where $\operatorname{deg}\left(v^{\prime}\right), \operatorname{deg}\left(v^{\prime \prime}\right) \geq m / 5, G$ contains a path of length at least $(1-5 \varepsilon) 2 m$ connecting $v^{\prime}$ and $v^{\prime \prime}$.

This lemma is used by Łuczak in [10]. Lemma 2 (with somewhat weaker parameters) also follows from the much stronger Blow-up Lemma (see [6] and [7]).

\section{FURTHER GRAPH THEORY TOOLS}

A set $M$ of pairwise disjoint edges of a graph $G$ is called a matching. The size $|M|$ of a maximum matching is the matching number, $v(G)$. A key notion in our approach is the notion of a connected matching. A matching $M$ is connected in $G$ if all edges of $M$ are in the same component of $G$. The following result is often referred to as the Tutte-Berge formula (see e.g., in [9] Theorem 3.1.14). We shall use $c(G)$ and $c_{o}(G)$ for the number of components and odd components of a graph $G$ and $\operatorname{def}(G)$, the deficiency of $G$, is defined as $|V(G)|-2 v(G)$.

Lemma 3. For any graph $G, \operatorname{def}(G)=\max \left\{c_{o}(G \backslash S)-|S|\right\}$ where the maximum is taken over all $S \subseteq V(G)$.

We also need the following obvious property of maximum matchings.

Lemma 4. Suppose $M=\left\{e_{1}, \ldots, e_{k}\right\}$ is a maximum matching in a graph $G$. Then $V(G) \backslash V(M)$ spans an independent set and one can select one end point $x_{i}$ of each $e_{i}$ so that for each $i, 1 \leq i \leq k$, there is at most one edge in $G$ from $x_{i}$ to $V(G) \backslash V(M)$.

For a tripartite graph $G=G(l, l, l)$, we shall work with its tripartite complement, $\bar{G}$, defined as the graph we obtain from the usual complement after deleting all edges within the partite classes. The next lemmas collect some simple properties of graphs of high density.

Lemma 5. Assume that $G=G(l, l, l)$ is a $(1-\varepsilon)$-dense tripartite graph. Then $G$ has a tripartite subgraph $H=H(k, k, k)$ with $k \geq(1-2 \sqrt{\varepsilon}) l$ such that: $A$. $\Delta(\bar{H})<2 \sqrt{\varepsilon} l ; B . \delta(H) \geq(2-6 \sqrt{\varepsilon}) l ; C$. H is $(1-3 \sqrt{\varepsilon})$-dense.

Proof. If $G$ has $p$ vertices in the same partite class with degree at least $2 \sqrt{\varepsilon} l$ in $\bar{G}$, then $\bar{G}$ has at least $p 2 \sqrt{\varepsilon} l$ edges. Therefore $p 2 \sqrt{\varepsilon} l \leq 3 \varepsilon l^{2}$, implying $p \leq$ $\frac{3}{2} \sqrt{\varepsilon} l<2 \sqrt{\varepsilon} l$. Removing these $p$ vertices from each partite class, the remaining vertices induce the subgraph $H$. Properties A. and B. are obvious and C. follows 
from

$$
|E(H)| \geq \frac{|V(H)| \delta(H)}{2} \geq \frac{|V(H)|(2-6 \sqrt{\varepsilon}) l}{2}=3 k(1-3 \sqrt{\varepsilon}) l \geq 3(1-3 \sqrt{\varepsilon}) k^{2} .
$$

Lemma 6. Assume $\Delta\left(\overline{G_{n}}\right)<\sqrt{\varepsilon}$ n and $H=[A, B]$ is a bipartite subgraph of $G_{n}$ with $2 \sqrt{\varepsilon} n<|A| \leq|B|$. Then $H$ is a connected subgraph of $G_{n}$ and contains a matching of size at least $|A|-\sqrt{\varepsilon} n$. Moreover, if only $2 \sqrt{\varepsilon} n<|B|$ and $A \neq \emptyset$ is assumed then there is a subgraph $H^{\prime}$ which is connected and covers $A$ and all but at most $\sqrt{\varepsilon} n$ vertices of $B$.

Proof. Two vertices in $A(B)$ have a common neighbor in $B(A)$. Also if $a \in$ $A, b \in B$ then any neighbor of $a$ and $b$ have a common neighbor in $A$. Thus $H$ is a connected subgraph. Moreover any maximum matching $M$ misses fewer than $\sqrt{\varepsilon} n$ vertices of $A$. The statement about $H^{\prime}$ follows by fixing a vertex $a \in A$ and $H^{\prime}$ is obtained by deleting from $B$ the vertices nonadjacent to $A$.

\section{LARGE MONOCHROMATIC CONNECTED MATCHINGS IN BALANCED TRIPARTITE GRAPHS}

A monochromatic (say red) matching in a colored complete or almost complete graph is called connected if its edges are all in the same monochromatic connected red component. For example, if $K_{4}$ is three-colored so that each color class has two disjoint edges (factorization of $K_{4}$ ) then the largest monochromatic matching has two edges, but the largest connected monochromatic matching has only one edge.

In our main lemma, we show that we can find large monochromatic connected matchings in balanced tripartite graphs.

Lemma 7. Suppose that $\sqrt{\varepsilon}<\frac{1}{132}$ and $l \geq \frac{1}{\sqrt{\varepsilon}(1-2 \sqrt{\varepsilon})}$. Then every two-coloring of a $(1-\varepsilon)$-dense tripartite graph $G(l, l, l)$ contains a monochromatic connected matching covering at least $(2-532 \sqrt{\varepsilon})$ l vertices.

Proof. By Lemma 5 select $H=H(k, k, k) \subseteq G(l, l, l)$ with $\Delta(\bar{H})<2 \sqrt{\varepsilon} l$, $\delta(H) \geq(2-6 \sqrt{\varepsilon}) l$ and $k \geq(1-2 \sqrt{\varepsilon}) l$. Let $V_{i}$ denote the partite classes of $H$.

We claim first that there is a set $Z \subseteq V(H)$ such that $|Z| \geq 3 k(1-6 \sqrt{\varepsilon})$ and in one of the two colors, the edges of this color inside $Z$ determine only one nontrivial component. This color is called the color of $Z$.

To prove the claim, select a largest monochromatic, say, red component $C_{1}$. Set $R_{i}=V_{i} \cap V\left(C_{1}\right), S_{i}=V_{i} \backslash V\left(C_{1}\right)$. If $V\left(C_{1}\right)$ covers two of the $V_{i}$-s then $Z=V(H)$ satisfies the claim with the red color. Thus at least two of the $S_{i}$-s are nonempty. Furthermore, by the choice of $C_{1},\left|C_{1}\right| \geq(1-3 \sqrt{\varepsilon}) l$ by B. of Lemma 5 .

Call a set small if it has less than $4 \sqrt{\varepsilon} l$ elements, otherwise it is large. The condition $\sqrt{\varepsilon}<\frac{1}{10}$ ensures that $8 \sqrt{\varepsilon} l \leq(1-2 \sqrt{\varepsilon}) l \leq k$, thus at least one of $\left|R_{i}\right|,\left|S_{i}\right|$ is large. Lemma 6 with $3 l$ in the role of $n$ and with $\frac{2}{3} \sqrt{\varepsilon}$ in the role of $\sqrt{\varepsilon}$ can be applied to $H$ since $\Delta(\bar{H})<2 \sqrt{\varepsilon} l=\frac{2}{3} \sqrt{\varepsilon} 3 l$. This gives that $\left[R_{i}, S_{j}\right]$ Journal of Graph Theory DOI 10.1002/jgt 
is connected in blue if both $R_{i}, S_{j}$ are large. Else it has a blue component covering all but at most $2 \sqrt{\varepsilon} l$ vertices of the larger part if only one of them is large and the other is nonempty. With these remarks in mind we have the following cases.

If all the three $S_{i}$-s are small then $C_{1}$ misses only these small sets, thus

$$
\left|C_{1}\right| \geq 3 k-12 \sqrt{\varepsilon} l \geq 3(1-2 \sqrt{\varepsilon}) l-12 \sqrt{\varepsilon} l=(1-6 \sqrt{\varepsilon}) 3 l \geq(1-6 \sqrt{\varepsilon}) 3 k
$$

and the claim follows with $Z=V\left(C_{1}\right)$ in color red.

If exactly one $S_{i}$, say, $S_{1}$ is large then $R_{2}, R_{3}$ are both large. Lemma 6 implies that $C_{2}=S_{1} \cup R_{2} \cup R_{3}$ is connected in blue. If $R_{1}$ is small then $C_{2}$ works as $Z$ in blue with the same estimate as (2). If $R_{1}$ is large then it is joined to $C_{2}$ through $S_{2}$ or through $S_{3}$, whichever is nonempty. Thus (2) works with reserve.

If exactly two $S_{i}$-s are large, say, $S_{1}, S_{2}$, then $S_{3}$ is small implying that $R_{3}$ is large. Lemma 6 ensures that $C_{3}=S_{1} \cup S_{2} \cup R_{3}$ is connected in blue. Then, applying Lemma 6 repeatedly, $R_{1}, R_{2}$ join to $C_{3}$. Thus $Z=V(H)$ works in color blue.

If all $S_{i}$-s are large we use that some $R_{i}$, say, $R_{1}$ is large,

$$
\left|R_{1}\right| \geq \frac{1}{3}\left|C_{1}\right| \geq \frac{1}{3}(1-3 \sqrt{\varepsilon}) l \geq 4 \sqrt{\varepsilon} l
$$

if $\sqrt{\varepsilon}<\frac{1}{15}$. Then, $R_{1} \cup S_{1} \cup S_{2} \cup S_{3}$ is connected in blue and $R_{2} \cup R_{3}$ is absorbed into that blue component. Thus, in this case $Z=V(H)$ is connected in blue and the claim is proved.

Now, we define a new tripartite graph $H^{\prime}$ by deleting all edges of $H$ inside $V(H) \backslash Z$ in the color of $Z$. Then we select a maximum monochromatic matching $M$ of $H^{\prime}$ in the color of $Z$, say, red, it is automatic that $M$ is connected. Apply Lemma 4 to select one end point of each edge of $M$, their set is denoted by $U$, the set of the other end points is denoted by $T$. Set $U_{i}=U \cap V_{i}, T_{i}=T \cap V_{i}$, let $M_{i j}$ denote the edges of $M$ going from $V_{i}$ to $V_{j}, m_{i j}=\left|M_{i j}\right|$. Set $W_{i}=V_{i} \backslash\left(U_{i} \cup T_{i}\right)$, define $H^{*}$ as the tripartite subgraph of $H^{\prime}$ induced by $V\left(H^{\prime}\right) \backslash\left(T_{1} \cup T_{2} \cup T_{3}\right)$.

Now Lemma 4 implies (with the convention that the exceptional red edge from each $u \in U_{i}$ to $W_{j}$ is deleted) that the following bipartite subgraphs of $H^{*}$ have only blue edges:

$$
\begin{aligned}
& {\left[U_{1}, W_{2}\right],\left[U_{1}, W_{3}\right],\left[U_{2}, W_{1}\right],\left[U_{2}, W_{3}\right],\left[U_{3}, W_{1}\right],\left[U_{3}, W_{2}\right],} \\
& {\left[W_{1}, W_{2}\right],\left[W_{1}, W_{3}\right],\left[W_{2}, W_{3}\right] .}
\end{aligned}
$$

From now on, we shall consider $H^{*}$ as the tripartite graph defined by the (blue) edge sets of the bipartite graphs in (3), thus the edge sets of the bipartite graphs $\left[U_{i}, U_{j}\right]$ are ignored.

Claim 1. Let $B$ be any of the bipartite graphs $\left[U_{i}, W_{j}\right],\left[W_{i}, W_{j}\right], i \neq j, 1 \leq i<$ $j \leq 3$. Then $\Delta(\bar{B})<22 \sqrt{\varepsilon} k$. 
Proof. Adding the losses from deleting the red edges of $V(H) \backslash Z$, one red edge per vertex from $u \in U_{i}$ to $W_{j}$ we have

$$
\begin{aligned}
\Delta(\bar{B}) & \leq 2 \sqrt{\varepsilon} l+(|V(H)|-|Z|)+1 \leq 2 \sqrt{\varepsilon} l+(3 k-(1-6 \sqrt{\varepsilon}) 3 k)+1 \\
& \leq\left(\frac{2 \sqrt{\varepsilon}}{1-2 \sqrt{\varepsilon}}+18 \sqrt{\varepsilon}\right) k+1<(3 \sqrt{\varepsilon}+18 \sqrt{\varepsilon}) k+1 \leq 22 \sqrt{\varepsilon} k
\end{aligned}
$$

where we used that $\sqrt{\varepsilon}<\frac{1}{6}$ and

proving Claim 1.

$$
\frac{1}{1-2 \sqrt{\varepsilon}} k \geq l \geq \frac{1}{\sqrt{\varepsilon}(1-2 \sqrt{\varepsilon})}
$$

Next we establish inequalities to prove that (the blue graph) $H^{*}$ has an almost spanning connected matching. Since each $V_{i}$ is partitioned by $U_{i}, T_{i}, W_{i}$ we have

$$
\left|U_{i}\right|+\left|T_{i}\right|+\left|W_{i}\right|=k
$$

for $1 \leq i \leq 3$. Also, we may assume

$$
|M|=\left|U_{1}\right|+\left|U_{2}\right|+\left|U_{3}\right|=\left|T_{1}\right|+\left|T_{2}\right|+\left|T_{3}\right|=m_{12}+m_{13}+m_{23}<k
$$

otherwise $M$ is a connected red matching of size $k \geq(1-2 \sqrt{\varepsilon}) l$, covering at least $(2-4 \sqrt{\varepsilon}) l$ vertices of $G$.

Notice that $\left|V\left(H^{*}\right)\right|=3 k-\left|T_{1} \cup T_{2} \cup T_{3}\right|=3 k-|M|>2 k$. Since $\left|W_{1}\right|+$ $\left|W_{2}\right|+\left|W_{3}\right|=3 k-2|M|>k$, (6) gives

$$
\sum_{i=1}^{3} U_{i}<\sum_{i=1}^{3} W_{i}
$$

Using (6),

$$
\begin{aligned}
& \left|U_{1}\right| \leq m_{12}+m_{13}<2 k-m_{12}-m_{13}-2 m_{23} \\
& \quad=k-\left(m_{12}+m_{23}\right)+k-\left(m_{13}+m_{23}\right)=\left|W_{2}\right|+\left|W_{3}\right|
\end{aligned}
$$

and by symmetry we get

$$
\left|U_{1}\right|<\left|W_{2}\right|+\left|W_{3}\right|,\left|U_{2}\right|<\left|W_{1}\right|+\left|W_{3}\right|,\left|U_{3}\right|<\left|W_{1}\right|+\left|W_{2}\right| .
$$

Since from (5) $\left|U_{1}\right|+\left|W_{1}\right|=k-\left|T_{1}\right|$ and $\left|U_{2}\right|+\left|W_{2}\right|+\left|U_{3}\right|+\left|W_{3}\right|=$ $\left|V\left(H^{*}\right)\right|-\left(\left|U_{1}\right|+\left|W_{1}\right|\right)$, using (6) we get the following

$$
\begin{aligned}
& \left(\left|U_{1}\right|+\left|W_{1}\right|\right)-\left(\left|U_{2}\right|+\left|U_{3}\right|+W_{2}|+| W_{3} \mid\right)=2 k-2\left|T_{1}\right|-\left|V\left(H^{*}\right)\right| \\
& \quad=\left|T_{2}\right|+\left|T_{3}\right|-\left|T_{1}\right|-k<0,
\end{aligned}
$$

Journal of Graph Theory DOI 10.1002/jgt 
giving the last set of inequalities:

$$
\begin{gathered}
\left|U_{1}\right|+\left|W_{1}\right|<\left|U_{2}\right|+\left|U_{3}\right|+\left|W_{2}\right|+\left|W_{3}\right|, \\
\left|U_{2}\right|+\left|W_{2}\right|<\left|U_{1}\right|+\left|U_{3}\right|+\left|W_{1}\right|+\left|W_{3}\right|, \\
\left|U_{3}\right|+\left|W_{3}\right|<\left|U_{1}\right|+\left|U_{2}\right|+\left|W_{1}\right|+\left|W_{2}\right| .
\end{gathered}
$$

Let $S$ be an arbitrary subset of $V\left(H^{*}\right)$. Partition $S$ into six parts, $S \cap U_{i}, S \cap W_{i}$ and let $S^{*}=S \cup M$ where $M$ is the union of those $U_{i}$-s and $W_{i}$-s that satisfy $\left|U_{i} \backslash S\right|<$ $44 \sqrt{\varepsilon} k$ or $\left|W_{i} \backslash S\right|<44 \sqrt{\varepsilon} k$. Then we have

$$
\left|S^{*}\right| \leq|S|+6 \times 44 \sqrt{\varepsilon} k .
$$

Claim 2. $c\left(H^{*} \backslash S^{*}\right) \leq\left|S^{*}\right|+1$.

Proof. Call $U_{i}\left(W_{i}\right)$ full, if $S^{*} \cap U_{i}=U_{i}\left(S^{*} \cap W_{i}=W_{i}\right)$. Observe that if $i \neq j$ and neither $U_{i}$ nor $W_{j}$ are full then $\left|U_{i} \backslash S^{*}\right| \geq 44 \sqrt{\varepsilon} k,\left|W_{j} \backslash S^{*}\right| \geq 44 \sqrt{\varepsilon} k$ and by Claim 1 at most $22 \sqrt{\varepsilon} k$ edges are missing from the bipartite graph $B=\left[U_{i} \backslash S^{*}, W_{j} \backslash S^{*}\right]$. Therefore, by Lemma $6, B$ is connected. The same argument shows that $\left[W_{i} \backslash S^{*}, W_{j} \backslash S^{*}\right]$ is connected for $i \neq j$ whenever $W_{i}, W_{j}$ are not full. This argument shows that there is at most one nontrivial component, all other components of $H^{*} \backslash S^{*}$ are trivial, that is, isolated vertices. It is obvious that removing vertices of $S^{*}$ from components that are not full can not decrease the number of components of $H^{*} \backslash S^{*}$. Therefore, we may assume all sets $U_{i}, W_{i}$ are either full or empty (i.e., $U_{i} \cap S^{*}, W_{i} \cap S^{*}$ are empty). This reduces the claim to check the following property of the weighted graph $Q$ on six vertices, the skeleton of $H^{*}$, defined with vertices $u_{i}, w_{i}, 1 \leq i \leq 3$ and edges $\left(u_{i}, w_{j}\right),\left(w_{i}, w_{j}\right), 1 \leq i<j \leq 3$ and vertex-weights $\left|U_{i}\right|,\left|W_{i}\right|:$

For every $S \subseteq V(Q)$ the total weight of the isolated points of $V(Q) \backslash S$ is smaller than the weight of $S$.

A moment of reflection gives that inequalities (7), (8), (9) state precisely this, finishing the proof of Claim 2.

Observe that for $X \subseteq X^{*}, c(G \backslash X)-|X| \leq c\left(G \backslash X^{*}\right)-\left|X^{*}\right|+2\left(\left|X^{*}\right|-|X|\right)$. Using this observation, Claim 2, (10), we get

$$
\begin{aligned}
c\left(H^{*} \backslash S\right)-|S| & \leq c\left(H^{*} \backslash S^{*}\right)-\left|S^{*}\right|+2\left(\left|S^{*}\right|-|S|\right) \leq 1+2\left(\left|S^{*}\right|-|S|\right) \\
& \leq 2 \times 6 \times 44 \sqrt{\varepsilon} k
\end{aligned}
$$

for any $S \subseteq V\left(H^{*}\right)$. Applying Lemma 3 we conclude that

$$
\begin{aligned}
& 2 k-2 v\left(H^{*}\right)<\left|V\left(H^{*}\right)\right|-2 v\left(H^{*}\right)=\operatorname{def}\left(H^{*}\right)=\max \left\{c_{o}\left(H^{*} \backslash S\right)-|S|\right\} \\
& \quad \leq \max \left\{c\left(H^{*} \backslash S\right)-|S|\right\} \leq 528 \sqrt{\varepsilon} k
\end{aligned}
$$

Journal of Graph Theory DOI 10.1002/jgt 
thus $H^{*}$ has a matching $M_{2}$ covering at least

$$
\begin{aligned}
& 2 k-528 \sqrt{\varepsilon} k \geq(2-528 \sqrt{\varepsilon})(1-2 \sqrt{\varepsilon}) l \\
& \quad=(2-532 \sqrt{\varepsilon}+1056 \varepsilon) l \geq(2-532 \sqrt{\varepsilon}) l
\end{aligned}
$$

vertices of $G$.

To see that $M_{2}$ is connected, we show that $H^{*}$ is connected. Recall that $\left|W_{1}\right|+$ $\left|W_{2}\right|+\left|W_{3}\right|>k$, so at least one $\left|W_{i}\right|$ is large, say, $\left|W_{1}\right| \geq \frac{k}{3}>44 \sqrt{\varepsilon} k$ if $\frac{1}{132}>\sqrt{\varepsilon}$. Also, by (6), all $W_{i}$-s are nonempty. Applying Lemma 6 to the bipartite graphs $\left[W_{2}, W_{1}\right],\left[W_{3}, W_{1}\right]$ we get components covering $W_{2}, W_{3}$ and all but at most $22 \sqrt{\varepsilon}$ vertices of $W_{1}$. These components must intersect by our assumption so $W_{1} \cup W_{2} \cup$ $W_{3}$ is in the same component $C$ of $H^{*}$. The same argument shows that $U_{2}, U_{3}$ also belongs to $C$. The only problematic set is $U_{1}$, which can be disconnected from $W_{2} \cup W_{3}$. To avoid that, we may assume that $|M|<(1-11 \sqrt{\varepsilon}) k$, otherwise the red matching is of size $(1-11 \sqrt{\varepsilon}) k \geq(1-11 \sqrt{\varepsilon})(1-2 \sqrt{\varepsilon}) l$, covering $(2-26 \sqrt{\varepsilon}+$ $44 \varepsilon) l>(2-26 \sqrt{\varepsilon}) l$ vertices of $G$. Then $\left|W_{2}\right|+\left|W_{3}\right|>2 k-2|M| \geq 2 k-2(1-$ $11 \sqrt{\varepsilon}$ ) $k=22 \sqrt{\varepsilon} k$ which (through Lemma 6) ensures that no vertex of $U_{1}$ can be disconnected from $C$.

It is easy to check that $2-532 \sqrt{\varepsilon}$ is the smallest coefficient of $l$ among the estimates.

\section{PROOF OF THEOREM 1}

We will assume that $n$ is sufficiently large. Let $0<\delta<1$ be arbitrary and choose

$$
\varepsilon=\frac{1}{3}\left(\frac{\delta}{225}\right)^{2}
$$

We need to show that each 2-edge coloring of $K(n, n, n)$ leads to a monochromatic path of length at least $(1-\delta) 2 n$. Consider a 2-edge coloring $\left(G_{1}, G_{2}\right)$ of $K(n, n, n)$. Let $V_{i}$ denote the partite classes. Apply the two-color tripartite version of the Regularity Lemma (Lemma 1), with $\varepsilon$ as in (11) and (by using the remark after the lemma) we can get a partition for $i=1,2,3$ of $V_{i}=V_{i}^{0}+V_{i}^{1}+\cdots+V_{i}^{l}$, where $\left|V_{i}^{j}\right|=m, 1 \leq j \leq l, 1 \leq i \leq 3$ and $\left|V_{i}^{0}\right|<\varepsilon n$, $1 \leq i \leq 3$. We define the following reduced graph $G^{r}$ : The vertices of $G^{r}$ are $p_{i}^{j}, 1 \leq j \leq l, 1 \leq i \leq 3$, and we have an edge between vertices $p_{i_{1}}^{j_{1}}$ and $p_{i_{2}}^{j_{2}}$, $1 \leq j_{1}, j_{2} \leq l, 1 \leq i_{1}, i_{2} \leq 3, i_{1} \neq i_{2}$, if the pair $\left\{V_{i_{1}}^{j_{1}}, V_{i_{2}}^{j_{2}}\right\}$ is $\left(\varepsilon, G_{s}\right)$-regular for $s=1,2$. Thus we have a one-to-one correspondence $f: p_{i}^{j} \rightarrow V_{i}^{j}$ between the vertices of $G^{r}$ and the non-exceptional clusters of the partition. Then $G^{r}$ is a $(1-\varepsilon)$-dense balanced tripartite graph. Define a 2-edge coloring $\left(G_{1}^{r}, G_{2}^{r}\right)$ of $G^{r}$ in the following way. The color of the edge between the clusters $V_{i_{1}}^{j_{1}}$ and $V_{i_{2}}^{j_{2}}$ is the majority color in the pair $\left\{V_{i_{1}}^{j_{1}}, V_{i_{2}}^{j_{2}}\right\}$. 
Lemma 7 implies that in such a 2-coloring of $G^{r}$ we can find a monochromatic connected matching $M=\left\{e_{1}, e_{2}, \ldots, e_{l_{1}}\right\}$ covering at least $(2-532 \sqrt{3 \varepsilon}) l$ vertices of $G^{r}$. Assume that $M$ is in $G_{1}^{r}$. Thus we have

$$
\left|\cup_{i=1}^{l_{1}} \cup_{p \in e_{i}} f(p)\right| \geq(2-532 \sqrt{3 \varepsilon})(1-\varepsilon) n \geq(2-534 \sqrt{3 \varepsilon}) n .
$$

Furthermore, define $f\left(e_{i}\right)=\left(C_{1}^{i}, C_{2}^{i}\right)$ for $1 \leq i \leq l_{1}$ where $C_{1}^{i}, C_{2}^{i}$ are the clusters assigned to the end points of $e_{i}$.

Since $M$ is a connected matching in $G_{1}^{r}$ we can find a connecting path $P_{i}^{r}$ in $G_{1}^{r}$ from $f^{-1}\left(C_{2}^{i}\right)$ to $f^{-1}\left(C_{1}^{i+1}\right)$ for every $1 \leq i \leq l_{1}-1$. Note that these paths in $G_{1}^{r}$ may not be internally vertex disjoint. From these paths $P_{i}^{r}$ in $G_{1}^{r}$, we can construct vertex disjoint connecting paths $P_{i}$ in $G_{1}$ connecting a typical vertex $v_{2}^{i}$ of $C_{2}^{i}$ to a typical vertex $v_{1}^{i+1}$ of $C_{1}^{i+1}$. More precisely, we construct $P_{1}$ with the following simple greedy strategy. Denote $P_{1}^{r}=\left(p_{1}, \ldots, p_{t}\right), 2 \leq t \leq 3 l$, where according to the definition $f\left(p_{1}\right)=C_{2}^{1}$ and $f\left(p_{t}\right)=C_{1}^{2}$. Let the first vertex $u_{1}\left(=v_{2}^{1}\right)$ of $P_{1}$ be a vertex $u_{1} \in C_{2}^{1}$ for which $\operatorname{deg}_{G_{1}}\left(u_{1}, f\left(p_{2}\right)\right) \geq m / 4$ and $\operatorname{deg}_{G_{1}}\left(u_{1}, C_{1}^{1}\right) \geq m / 4$. By $\varepsilon$-regularity, most of the vertices satisfy this in $C_{2}^{1}$. The second vertex $u_{2}$ of $P_{1}$ is a vertex $u_{2} \in\left(f\left(p_{2}\right) \cap N_{G_{1}}\left(u_{1}\right)\right)$ for which $\operatorname{deg}_{G_{1}}\left(u_{2}, f\left(p_{3}\right)\right) \geq m / 4$. Again by regularity most vertices satisfy this in $f\left(p_{2}\right) \cap N_{G_{1}}\left(u_{1}\right)$. The third vertex $u_{3}$ of $P_{1}$ is a vertex $u_{3} \in\left(f\left(p_{3}\right) \cap N_{G_{1}}\left(u_{2}\right)\right)$ for which $\operatorname{deg}_{G_{1}}\left(u_{3}, f\left(p_{4}\right)\right) \geq m / 4$. We continue in this fashion, finally the last vertex $u_{t}\left(=v_{1}^{2}\right)$ of $P_{1}$ is a vertex $u_{t} \in$ $\left(f\left(p_{t}\right) \cap N_{G_{1}}\left(u_{t-1}\right)\right)$ for which $\operatorname{deg}_{G_{1}}\left(u_{t}, C_{2}^{2}\right) \geq m / 4$.

Then we move on to the next connecting path $P_{2}$. Here we follow the same greedy procedure, we pick the next vertex from the next cluster in $P_{2}^{r}$. However, if the cluster has occurred already on the paths $P_{1}^{r}$ or $P_{2}^{r}$, then we just have to make sure that we pick a vertex that has not been used on $P_{1}$ or $P_{2}$.

We continue in this fashion and construct the vertex disjoint connecting paths $P_{i}$ in $G_{1}, 1 \leq i \leq l_{1}-1$. These will be parts of the final path in $G_{1}$. We remove the internal vertices of these paths from $G_{1}$. By doing this we may create some discrepancies in the cardinalities of the clusters of this connected matching. We remove at most $(3 l)^{2}$ vertices from each cluster of the matching to assure that now we have the same number of vertices left in each cluster of the matching. Then by applying Lemma 2 for $1 \leq i \leq l_{1}$, we get a path in $\left.G_{1}\right|_{f\left(e_{i}\right)}$ connecting $v_{1}^{i}$ and $v_{2}^{i}$ that contains almost all of the vertices of $f\left(e_{i}\right)$ (in case of $i=1$ we just select a long path of $f\left(e_{1}\right)$ starting from $v_{2}^{1}$ and in case of $i=l_{1}$, we select a long path of $f\left(e_{l_{1}}\right)$ starting from $v_{1}^{l_{1}}$. Finally using (11) and (12) we get a path in $G_{1}$ that contains at least

$$
(2-534 \sqrt{3 \varepsilon}-16 \varepsilon) n \geq(2-550 \sqrt{3 \varepsilon}) n=(1-225 \sqrt{3 \varepsilon}) 2 n=(1-\delta) 2 n
$$

vertices. This completes the proof of Theorem 1. 


\section{ACKNOWLEDGEMENTS}

This Research was Supported in part by OTKA grants, T038198 and T046234. and also in part by the National Science Foundation under grant no. DMS-0456401.

\section{REFERENCES}

[1] B. Bollobás, Extremal Graph Theory, Academic Press London, 1978.

[2] A. Figaj and T. Łuczak, The Ramsey number for a triple of long even cycles, J Combin Theory (to appear).

[3] L. Gerencsér and A. Gyárfás, On Ramsey-type problems, Ann Univ Sci Budapest Eötvös Sect Math 10 (1967), 167-170.

[4] R. L. Graham, B. L. Rothschild, and J. H. Spencer, Ramsey Theory, John Wiley \& Sons, New York, 1990.

[5] A. Gyárfás, G. N. Sárközy, E. Szemerédi, and M. Ruszinkó, Three-color Ramsey numbers for paths, Combinatorica 27(1) (2007), 35-69.

[6] J. Komlós, G. N. Sárközy, and E. Szemerédi, Blow-up Lemma, Combinatorica 17 (1997), 109-123.

[7] J. Komlós, G. N. Sárközy, and E. Szemerédi, An algorithmic version of the Blow-up Lemma, Random Struct Algo 12 (1998), 297-312.

[8] J. Komlós and M. Simonovits, Szemerédi's Regularity Lemma and its applications in graph theory, Combinatorics, Paul Erdős is Eighty, D. Miklós, V. T. Sós, and T. Szônyi (Editors), Bolyai Society Math Studies, Budapest, 1996, Vol. 2, pp. 295-352.

[9] L. Lovász and M. D. Plummer, Matching Theory, Joint edition of NorthHolland and Akadémiai Kiadó, 1986.

[10] T. Łuczak, $R\left(C_{n}, C_{n}, C_{n}\right) \leq(4+o(1)) n$, J Combin Theory, Ser B 75 (1999), 174-187.

[11] C. Magyar and R. R. Martin, Tripartite version of the Corrádi-Hajnal theorem, Discrete Math 254 (2002), 289-308.

[12] S. P. Radziszowski, Small Ramsey numbers, Elect J Combin, (2002) DS1.

[13] F. P. Ramsey, On a problem of formal logic, Proc London Math Soc, 2nd Ser, 30 (1930), 264-286.

[14] E. Szemerédi, Regular partitions of graphs, Colloques Internationaux C.N.R.S. № 260, Problèmes Combinatoires et Théorie des Graphes, Orsay (1976), 399401. 